\title{
Análisis experimental y numérico del método del intercepto para evaluar la extensión estable de grieta en una probeta $\mathrm{C}(\mathrm{T})$ de $\operatorname{acero}^{(\bullet)}$
}

\author{
J. R. Donoso*, R. Leiva** y F. Labbé**
}

\begin{abstract}
Resumen
Se presenta un nuevo método de obtención del tamaño de grieta para un ensayo fractomecánico, cuando se tiene sólo información fuerza-desplazamiento, $P$-v. Este método consiste en generar curvas $P-v$ de tipo "a constante", y evaluar sus interceptos con la curva experimental. Se demuestra que el método contribuye a generar información fidedigna respecto de la evolución del tamaño de grieta en el ensayo, usando datos $P$ - $v$-a de una probeta $C(T)$ de acero A 508 y con el respaldo de una modelación bidimensional por elementos finitos. Los resultados obtenidos con el método permiten generar una curva $J-R$ prácticamente idéntica a aquella construida a partir de los datos experimentales originales.
\end{abstract}

Palabras clave

Extensión estable de grieta; Formato común; Formato conciso; Fractura elasto-plástica; Ley de crecimiento de grieta.

\section{Experimental and numerical analysis of the intercept method for evaluating stable crack extension in a steel $\mathrm{C}(\mathrm{T})$ specimen}

\begin{abstract}
A new method for evaluating the crack size in a fracture mechanics test which only has force-displacement data, $P$ $v$, is introduced. This method consists in generating P-v curves of the "a constant" type, and obtain their intercepts with the experimental curve. By using P-v-a data of an A $508 \mathrm{C}(\mathrm{T})$ specimen, and a two-dimensional finite element mod$\mathrm{el}$, it is shown that the method contributes to generate relevant data concerning the evolution of the crack size in a test. The results obtained with the method make it possible to construct a $J-R$ curve which is practically identical to that constructed with the original experimental data.
\end{abstract}

Keywords

Stable crack growth; Common format; Concise format; Elastic-plastic fracture; Crack growth law.

\section{INTRODUCCIÓN}

La tenacidad a la fractura de un material dúctil se puede evaluar, de acuerdo a la Norma E $1820^{[1]}$, como un valor puntual, provisionalmente designado como $J_{Q}$, que puede convertirse en $J_{I c}$, siempre que se cumplan ciertos requisitos de tamaño. En un artículo reciente, Pehrson y Landes ${ }^{[2]}$ demostraron, empíricamente, que $J_{\mathrm{Q}}$, el valor puntual de la tenacidad a la fractura, correlaciona bien con el valor de $J$ evaluado a carga máxima para probetas $1 \mathrm{~T}-\mathrm{C}(\mathrm{T})$, de ancho $W=50 \mathrm{~mm}$; es decir, $J_{Q} \approx J\left(P_{\max }\right)$. En este contexto, Pehrson y Landes sugieren que $J_{I c}$ se puede estimar como $J$ a $0,99 P_{\max }$, con un alto grado de certeza estadística. Aunque el valor de $J_{\mathrm{O}}$ depende del tamaño (en este caso, de $W$ ), los resultados de Pehrson y Landes demuestran que se puede realizar un ensayo de fractura, analizar los datos generados fuerza-desplazamiento $(P-v)$ y, a partir de éstos, obtener una estimación razonable sólo para la propiedad del material $J_{I c}$, relacionada con el comienzo del proceso de extensión estable de la grieta.

De otra parte, la Norma E 1820 establece que la tenacidad a la fractura también se puede obtener como una curva de resistencia J (la denominada curva $J-R$ ), a partir de un ensayo de fractura de una probeta única. Puesto que la construcción de la

\footnotetext{
(•) Trabajo recibido el día 12 de marzo de 2008 y aceptado en su forma final el día 14 de abril de 2009.

* Profesor, Departamento Ciencia de Materiales, Universidad Santa María, Valparaíso, CHILE; juan.donoso@usm.cl

** Alumno de Postgrado y Profesor, Departamento de Mecánica, Universidad Santa María, Valparaíso, CHILE.
} 
curva $J-R$ implica considerar el proceso de extensión estable de grieta y, con él, alguna forma de corrección incremental de $J$ debido al cambio en tamaño de grieta, el tamaño real de ésta se debe medir simultáneamente con el ensayo, por medio de cambios en la flexibilidad elástica o por otras técnicas. De acuerdo con esto, la información fundamental para ensayos conducentes a generar este tipo de curva es: fuerza $(P)$, desplazamiento del punto de carga $(v)$ y tamaño de grieta $(a)$. Así, es esencial contar con el valor actualizado de extensión de grieta, $\Delta a_{i}=a_{i}-a_{o}$, donde $a_{i}$ es el tamaño de grieta correspondiente al punto " $i$ " del registro $P-v$ y $a_{0}$ es el tamaño de grieta inicial, para realizar la construcción de la curva $J-R$.

Cuando se tiene el registro $P-v$ de un ensayo de fractura, pero sólo los tamaños de grieta inicial y final, medidos a posteriori sobre la superficie de fractura, la Norma E 1820 permite obtener la curva J-R a través de la técnica de "normalización". Esta metodología alternativa (que aparece explicada en el Anexo A15 de E 1820) es utilizable sólo bajo ciertas condiciones establecidas en la Norma y permite generar valores de tamaño de grieta $a_{i}\left(\operatorname{con} a_{o}<a_{i}<a_{f}\right)$ sin haberlas medido directamente en el transcurso del ensayo. En este contexto, han aparecido nuevos métodos para evaluar tamaño de grieta para cada punto de la curva cuando se tiene sólo los datos $P-v$ y los tamaños de grieta inicial y final: la noción de la "ley de crecimiento de grieta", postulada por Donoso, Zahr y Landes (DZL $)^{[3 \text { y } 4]} \mathrm{y}$, recientemente, el denominado "método del intercepto" [5]. Estos métodos presentan algunas ventajas, cuando en un ensayo de fractura no es posible usar el método de la flexibilidad, y representan una alternativa al método de normalización incluido en E 1820.

En este trabajo se presenta el método del intercepto, y se aplica al caso de una probeta $\mathrm{C}(\mathrm{T})$ de acero ASTM A 508, de la cual se tiene el registro $P-v-a$, generando así la ley de crecimiento de grieta que permite determinar la evolución de su tamaño en el ensayo. A partir de los valores de extensión de grieta, tanto experimentales como calculados por el método, se puede construir la curva J-R para la probeta. Luego, la hipótesis sobre la cual se basa el método del intercepto es verificada mediante una modelación numérica bidimensional de la probeta $\mathrm{C}(\mathrm{T})$, utilizando la ley de crecimiento obtenida para emular un ensayo de fractura con crecimiento estable de grieta por el método de elementos finitos.

En lo que sigue, se muestran los fundamentos de la ley de crecimiento de grieta, el método del intercepto aplicado a un caso real, la modelación numérica que da sustento al método y la curva $J-R$ construida con los datos aportados por el método.

\section{EL MODELO DZL DE CRECIMIENTO DE GRIETA}

Un ensayo fractomecánico de un material dúctil se lleva, normalmente, a desplazamientos mayores que aquél correspondiente a la fuerza máxima. La figura 1 muestra una curva $P-v$ esquemática que presenta una fuerza máxima $P_{\max }$. Esta curva se denomina como "a variable", para reflejar el hecho de que una probeta que presenta tal curva experimenta una extensión estable de fisura. El ensayo se termina, intencionalmente, en el punto designado como " $F$ ", representado por la tríada " $P_{f}, v_{f}, a_{f}$ ", indicando, así, que la fuerza y el desplazamiento en el punto final del ensayo son $\left(P_{f}, v_{f}\right)$ y el tamaño de grieta es $a_{f}$. La cuantía de crecimiento estable de grieta al final del ensayo de fractura es $\Delta a=a_{f}-a_{0}$.

En la curva "a variable", la fuerza aumenta hasta el valor $P_{\max }$ y, luego, disminuye. La fuerza máxima se alcanza para el desplazamiento denominado " $v_{c}$ "; así, el valor de $J_{Q}$ (esto es, $J$ para $P_{\max }$ ) fue calculado por Pehrson y Landes como ${ }^{[2}$ 6]:

$$
J_{Q}=\frac{m}{B b} \int_{0}^{v_{\varepsilon}} P d v
$$

En la ecuación (1), m es el factor "eta" plástico, $B$ es el espesor y $b$, el ligamento resistente, igual a $W-a$.

Existe una segunda curva que intercepta a la primera, designada como "a constante". Esta es la curva

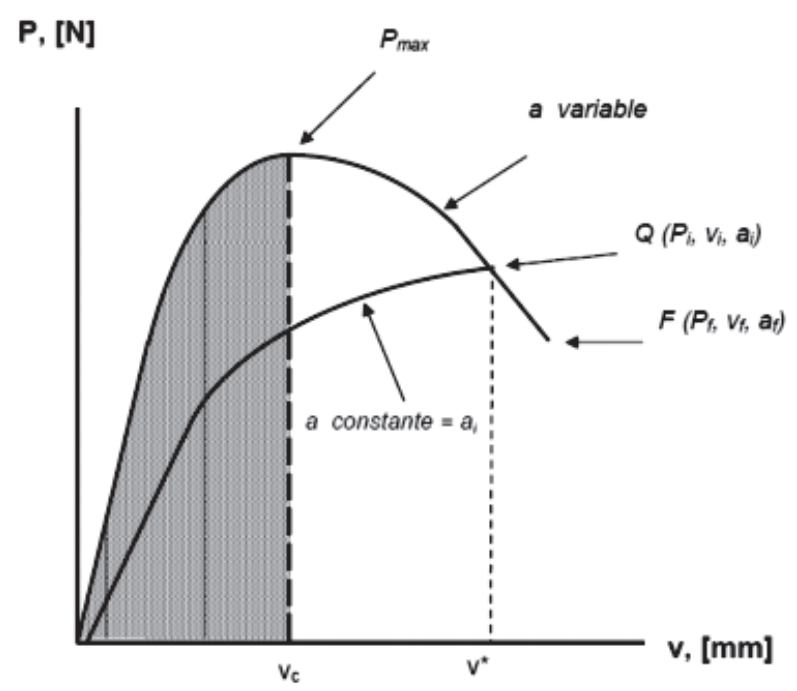

Figura 1. Curvas $P$-v esquemáticas de tipo "a variable" y "a constante".

Figure 1. Schematic P-v curves of the "a variable" and "a constant" type. 
que la probeta mostraría en ausencia de crecimiento de la grieta, es decir, representa el comportamiento de una probeta con entalla roma, de tamaño inicial de grieta $a_{i}$, el cual, idealmente, no cambia. Para esta situación, la fuerza va constantemente en aumento, siguiendo un patrón de comportamiento bien conocido, dado por la conjunción del Formato Común ${ }^{[7]}$ y el Formato Conciso ${ }^{[8]}$.

Donoso, Zahr y Landes ${ }^{[3 \text { y } 4]}$ (DZL), propusieron una manera alternativa y novedosa de obtener la curva $J-R$ usando los Formatos Común ${ }^{[7]}$ y Conciso ${ }^{[8]}$ (designados en adelante $C \& C$ ). Entre muchas ventajas, el uso de estos formatos ha hecho posible relacionar la fuerza máxima en una curva $P-v$ con los valores de la extensión de la grieta, para una probeta que experimenta un crecimiento estable de la grieta $^{[9]}$. Las relaciones $C \& C$ requeridas para este análisis se presentan a continuación.

Los Formatos Común y Conciso desarrollados por Donoso y Landes son funciones de calibración que relacionan la carga, $P$, con el desplazamiento, $v$, y la longitud de la grieta, $a$, de una probeta fractomecánica. El Formato Común tiene que ver con la componente plástica del desplazamiento, $v_{p l}{ }^{[7]}$, mientras que el Formato Conciso representa el régimen elástico, en el cual $v=v_{e l}{ }^{[8]}$. Estas relaciones $P-v-a$ se basan en el concepto de separación de la fuerza, $P$, en una función dependiente de la geometría y en una función dependiente de la deformación ${ }^{[10]}$. La función geométrica depende del tamaño de la grieta y lo hace a través de una relación de ley de potencia en el tamaño normalizado del ligamento, $b / W$, donde, $W$ es el ancho de la probeta. La función de deformación, por otro lado, depende de $v_{p l} / W$ a través de una relación de ley de potencia en el Formato Común y, linealmente, de $v_{e l} / W$, en el Formato Conciso. Ya que una probeta de ensayo que experimenta una extensión estable de grieta muestra una gran cantidad de desplazamiento plástico comparado con el elástico, el análisis se puede realizar usando, sólo, el Formato Común. La Ecuación del Formato Común (EFC) incluye además un término que denota la constricción fuera-del-plano, $\Omega^{*}$, y se escribe como:

$$
P=\Omega^{*} \cdot B \cdot C \cdot W \cdot(b / W)^{m} \cdot \sigma^{*} \cdot(v p l / W)^{1 / n}
$$

donde, $B$ es el espesor de la probeta; $C$ y $m$ son los parámetros de la función geométrica y $\sigma^{*}$ y $n$ son propiedades del material, las que se puede obtener de la curva tensión-deformación del material ${ }^{[7]} \mathrm{O}$, directamente, de la curva fuerza normalizada-desplazamiento plástico normalizado de la probeta ${ }^{[4}$ y 11].

Para una probeta con grieta estacionaria, el tamaño de la grieta (o ligamento) es constante y $P$ y $v$ se convierten en variables de la función de calibración, para tamaño de grieta constante. Sin embargo, cuando existe extensión estable de grieta, el tamaño de la grieta, $a$, también se convierte en variable, de manera que se necesita una relación separada entre $a$ y $v_{p l}$. Para tal propósito, Donoso et al. propusieron una "ley de crecimiento de la grieta" [3 y ${ }^{4]}$ para justificar la relación entre la cuantía de extensión estable, $\Delta a$, y el desplazamiento plástico, $v_{p l}$. La ley de crecimiento de grieta asumida es una ecuación de ley de potencia, con dos parámetros, que relaciona el cambio en el tamaño normalizado de grieta, $\Delta a / W$, con el desplazamiento plástico normalizado, $v p l / W$; es decir,

$$
\frac{\Delta a}{W}=I_{0}\left(\frac{v_{p l}}{W}\right)^{I_{1}}
$$

En la ecuación (3), $l_{o}$ es un coeficiente a determinar (como se explicará luego) y $l_{1}$, un exponente, el cual es del orden de $2,0^{[3]}$ para probetas $\mathrm{C}(\mathrm{T})$.

El término $\Delta a$, extensión de la grieta, además, se puede escribir en función del cambio en el tamaño del ligamento, esto es, $\Delta a=b_{o}-b$, donde $b_{o}$ es el tamaño inicial del ligamento $\left(b_{0}\right.$ es igual a $W$ menos el tamaño inicial de la grieta, $\left.a_{o}\right)$. De esta forma, la ecuación (3) da la siguiente expresión para el tamaño actual del ligamento, $b$ :

$$
\frac{b}{W}=\frac{b_{o}}{W}-I_{\circ}\left(\frac{v_{p l}}{W}\right)^{I_{1}}
$$

La sustitución de la ecuación (4) en la ecuación (2) arroja la siguiente expresión para la EFC, sólo, en función del desplazamiento plástico cuando existe crecimiento estable de la grieta:

$$
P=D B C W\left(\frac{b_{o}}{W}-I_{\circ}\left(\frac{v_{p l}}{W}\right)^{I_{1}}\right)^{m}\left(\frac{v_{p l}}{W}\right)^{1 / n}
$$

donde, $D$ es el producto de los parámetros $\sigma^{*}$ y $\Omega^{*}$.

De otra parte, la sustitución de las Ecs. (3) y (4) en la Ec. (2), da la EFC para una situación de crecimiento de la grieta sólo en función de la extensión de la grieta, $\Delta a$ :

$$
P=D B C W\left[\frac{b_{o}-\Delta a}{W}\right]^{m}\left(\frac{1}{I_{0}} \frac{\Delta a}{W}\right)^{1 /(n / 1)}
$$


La ecuación (5) es la relación del modelo de DZL para la curva fuerza, vs, desplazamiento plástico cuando hay crecimiento estable de grieta. La ecuación (6), de otra parte, muestra solamente la relación entre fuerza y tamaño de grieta, cuando existe crecimiento estable de grieta. La forma de ambas relaciones indica, claramente, la existencia de un valor máximo para la fuerza $P$, en función del desplazamiento plástico (Ec. (5)) o del tamaño de grieta (Ec. (6)).

El procedimiento definido en la Norma E 1820 para la construcción de la curva $J-R$, señala que se debe contar con el registro fuerza, vs. desplazamiento total y con los datos de la extensión estable de la grieta, en función del desplazamiento total, como un complemento del resultado $P-v$. La mirada alternativa a la construcción de la curva $J-R$ propuesta por DZL, incluye la ley de crecimiento de la grieta, (Ec. (3)), y la relación entre fuerza y desplazamiento plástico para una situación de crecimiento de grieta (Ec. (5)). Ambas ecuaciones (Ec. (3) y Ec. (5)) relacionan $\triangle a$ y $P$ con el componente plástico del desplazamiento; el desplazamiento elástico, por otra parte, se puede obtener con el uso del Formato Conciso ${ }^{[8]}$.

\section{UN MODELO ALTERNATIVO DE CRECIMIENTO DE LA GRIETA PARA UNA PROBETA C(T)}

El modelo original DZL usó un valor del exponente $l_{1}$ de la ley de crecimiento de la grieta, (Ec. (3)), igual a 2,0 para los datos de las probetas $\mathrm{C}(\mathrm{T})$ examinadas ${ }^{[3}$ y 4$]$. De otra parte, el valor del coeficiente $l_{o}$ se obtuvo por calibración con el punto final del ensayo. Usando la notación de la figura 1, se emplearon los valores del conjunto " $P_{f}, v_{f}, a_{f}$ " para obtener $l_{o}$ como:

$$
I_{o}=\left(\frac{\Delta a_{f}}{W}\right)\left(\frac{v_{p l, f}}{W}\right)^{-I_{1}}
$$

donde, el componente plástico de la ecuación (7) al final del ensayo, $v_{p l, f}$, es:

$$
v_{p l, f}=v_{f}-v_{e l, f}
$$

y el desplazamiento elástico, $v_{e l, f}$, es:

$$
v_{e l, f}=c_{f} P_{f}
$$

En la ecuación (9), $c_{f}$ es la flexibilidad elástica (una función del valor final del tamaño de grieta, $a_{f}$ ) en el punto final del ensayo. La flexibilidad elástica, para un tamaño dado de grieta, $a$, se calcula a partir de las ecuaciones disponibles en el Apéndice A12 de $\mathrm{E} 1820^{[1]}$.

La figura 2 muestra cuatro curvas $P$-v de una probeta 1T-C(T) de acero ASTM A 508, para la cual, $B$ $=25 \mathrm{~mm}, W=50 \mathrm{~mm}$, y los tamaños de grieta inicial y final son $a_{o}=26,2 \mathrm{~mm}$ y $a_{f}=33,1 \mathrm{~mm}$, respectivamente. En la figura 2, los puntos sólidos $(\bullet)$ unidos por la línea continua corresponden a los datos experimentales de origen. Adicionalmente, se muestran tres curvas generadas utilizando los Formatos $C \& C$; una, para "a variable", siendo coincidente con la curva experimental A 508 y, otras dos, del tipo "a constante" ${ }^{[4} \mathrm{y}^{5]}$. Estas últimas dos curvas fueron construidas sobre la base de la ecuación (2), y con tamaños de grieta inicial, $a_{o}$ (curva superior), y final, $a_{f}$ (curva inferior). Los parámetros usados son $D=\Omega * \sigma=372$ $\mathrm{MPa}, \mathrm{y} n=5,25^{[3 \text { y } 4]}$. La curva "a variable" se caracteriza por los mismos valores de $D$ y $n$ pero, además, por los parámetros $l_{o} \approx 52$ y $l_{1}=2,0$. El valor de $l_{o}$ se calcula, como se señaló antes, a partir de $l_{1}$, con los datos " $P_{f}, v_{f}, a_{f}$ " y con las ecuaciones (7), (8) y (9).

Es importante notar que la curva experimental fue construida con un número discreto de puntos: 22 en total, en los cuales se conocía conjuntamente fuerza, desplazamiento, y tamaño de grieta. En cambio, las tres curvas $\mathrm{C} \& \mathrm{C}$ (las dos a constante y la a variable) fueron construidas con un elevado número de puntos, dado el carácter analítico de los Formatos C\&C.

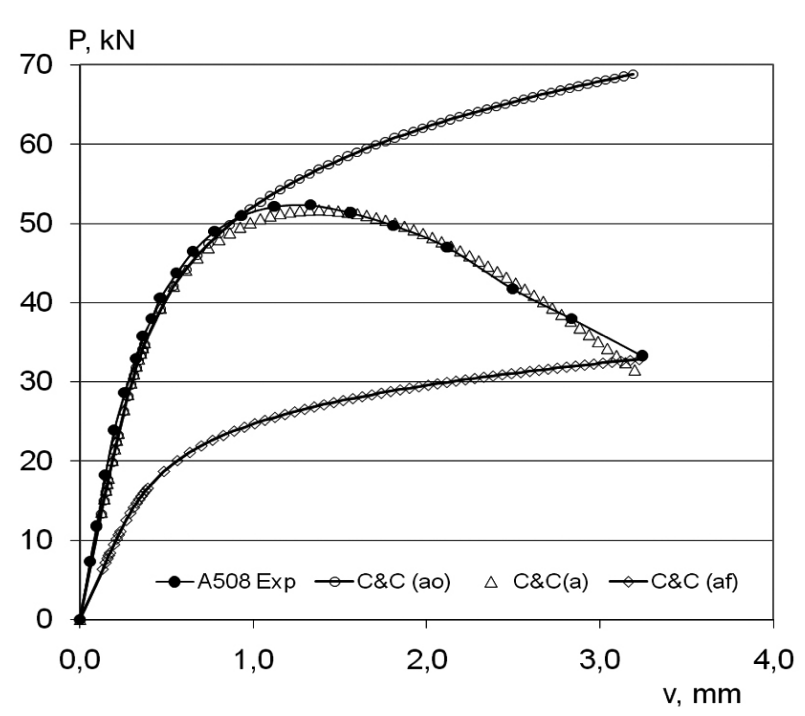

Figura 2. Curvas $P$-v para la probeta $C(T)$ de acero ASTM A 508. Se muestran la curva experimental (A508 Exp) y tres curvas obtenidas utilizando los formatos C\&C.

Figure 2. P-v curves for the ASTM A 508 steel $C(T)$ specimen. The experimental curve is shown (A508 Exp) together with three curves obtained with the C\&C formats. 
Para introducir el método del intercepto, considérese la figura 3 para la probeta de A 508. Esta figura incluye la curva $P-v$ experimental y una curva " $a$ constante", construida con el valor de tamaño de grieta denominado "18": el punto " $F$ " (con referencia a la figura 1) es el punto experimental número 22, y el "Q", el 18.

La figura 3 muestra las dos curvas, interceptándose en un punto cercano a un valor de fuerza de 50 $\mathrm{kN}$. El desplazamiento total correspondiente se ha denominado como $v^{*}$. Para la curva experimental, entonces, rige la siguiente relación:

$$
v^{*}=v_{x, e l}+v_{x, p l}
$$

donde, "x" simboliza el hecho de que el tamaño de grieta es desconocido en ese punto y deberá ser determinado.

De otra parte, para la curva "a constante" con tamaño de grieta $a_{18}$, se tiene:

$$
v^{*}=v_{18, e l}+v_{18, p l}
$$

Los dos desplazamientos deben ser iguales en el punto de intersección, por lo que:

$$
v_{x, e l}+v_{x, p l}=v_{18, e l}+v_{18, p l}
$$

En el punto de intersección, la curva "18" tiene un desplazamiento elástico dado por la fuerza en ese

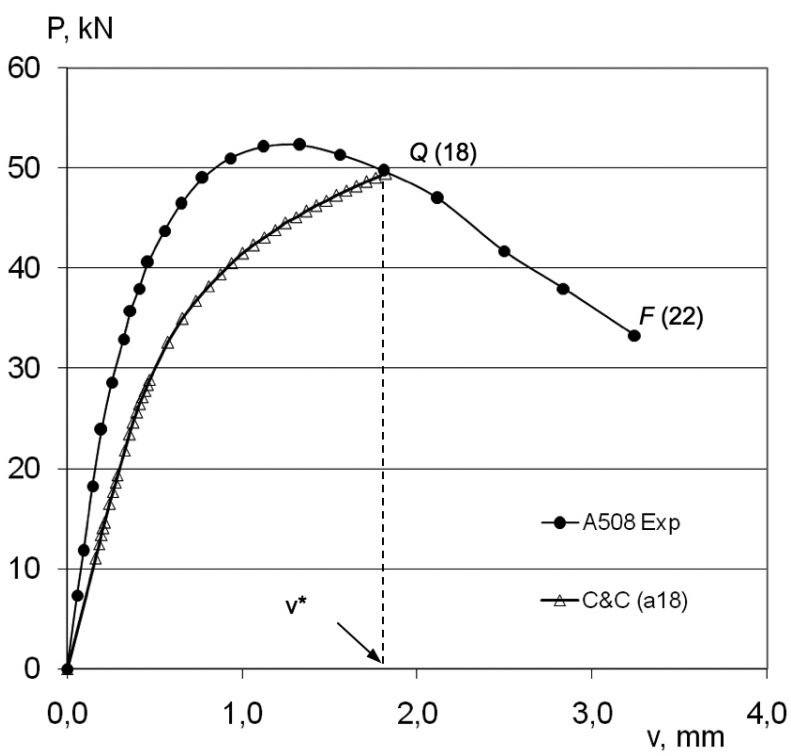

Figura 3. Curva $P$-v experimental de A 508 con una curva del tipo "a constante" superpuesta.

Figure 3. Experimental A $508 P$-v curve with one "a constant" curve overlapped. punto $(50 \mathrm{kN})$ y por la flexibilidad elástica correspondiente al tamaño de grieta " 18 ". En ese mismo punto, la curva experimental A 508, que en el punto de intersección tiene un tamaño de grieta de valor " 18 ", deberá, entonces, tener un desplazamiento elástico igual al desplazamiento elástico de la curva $C \& C$.

De hecho, si el tamaño de grieta " 18 " se ha medido experimentalmente en la probeta A 508 mediante el método de la flexibilidad en descarga, entonces, la pendiente de la línea de descarga en el punto Q debería ser igual a la pendiente elástica inicial de la curva "a constante" 18. Luego, si $v_{x, e l}=v_{18, e l}$, entonces se cumple:

$$
v_{x, p l}=v_{18, p l}
$$

La ecuación (13) indica que los desplazamientos plásticos en el punto de intersección son iguales, sin importar cómo las dos curvas lleguen a ese punto: la curva experimental, que sigue una trayectoria de carga (deformación y crecimiento estable de grieta) y la curva "a constante", que sigue una trayectoria de sólo deformación, sin crecimiento de grieta. Esto es muy relevante para la curva experimental ya que la trayectoria que debe seguir para alcanzar el punto "18" es el resultado de una competencia entre un proceso de endurecimiento por deformación y un proceso de extensión estable de la grieta.

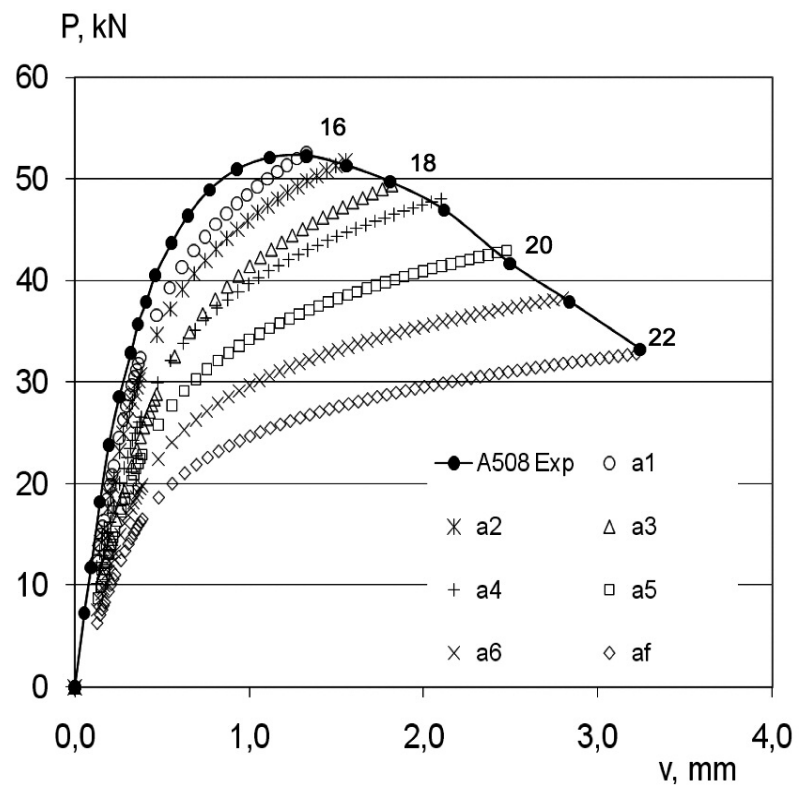

Figura 4. Aplicación del método a la curva $P-v$ de la probeta $\mathrm{C}(\mathrm{T})$ de $\mathrm{A} 508$.

Figure 4. Application of the method to the $A$ $508 C(T)$ specimen P-v curve. 
La figura 4 muestra, gráficamente, siete curvas del tipo "a constante", construidas con valores de $a$ entre $a_{1}$ y $a_{f}$. Los valores de tamaño de grieta corresponden a aquellos medidos en el ensayo por la técnica de la flexibilidad en descarga, para cada punto, desde el " 16 " hasta el "22". En la figura 4 se señalan estos puntos, correspondiendo el " 16 " a $a_{1}$ y el " 22 " a $a_{f}$. Dentro de cierto margen de error, las siete curvas $C \& C$ interceptan a la curva experimental en, o cerca de, cada uno de los puntos denominados 16 a 22 y caracterizados por un valor de tamaño de grieta entre $a_{1}$ y $a_{f}$.

El resultado de este procedimiento se muestra en la figura 5, en la forma tamaño de la grieta vs. desplazamiento. Los símbolos abiertos (o) corresponden a los resultados del método aplicado a los tamaños de grieta propios de los puntos experimentales " 16 " al "22", en tanto que los valores del desplazamiento son los del intercepto entre la curva experimental y cada una de las curvas $C \& C$ construidas con los valores de tamaño de grieta entre $a_{1} \approx 27 \mathrm{~mm}$ y $a_{\mathrm{f}}$. La curva continua, en tanto, corresponde a los datos experimentales originales. En esta figura se puede advertir que el método da valores $a-v$ prácticamente idénticos a los experimentalmente medidos.

La figura 6 muestra la ley de crecimiento, en la típica forma $\Delta a / W$ vs $v_{p l} / W$, para los valores obtenidos con el método del intercepto. En esta ocasión, los parámetros de la ecuación (3) son $l_{o} \approx 44$ y $l_{1} \approx 1,91$. Estos, deben ser comparados con los valores obtenidos con los datos experimentales, citados previamen$\mathrm{te}^{[5]}$, de $l_{0} \approx 52$ y $l_{1}=2,0$.

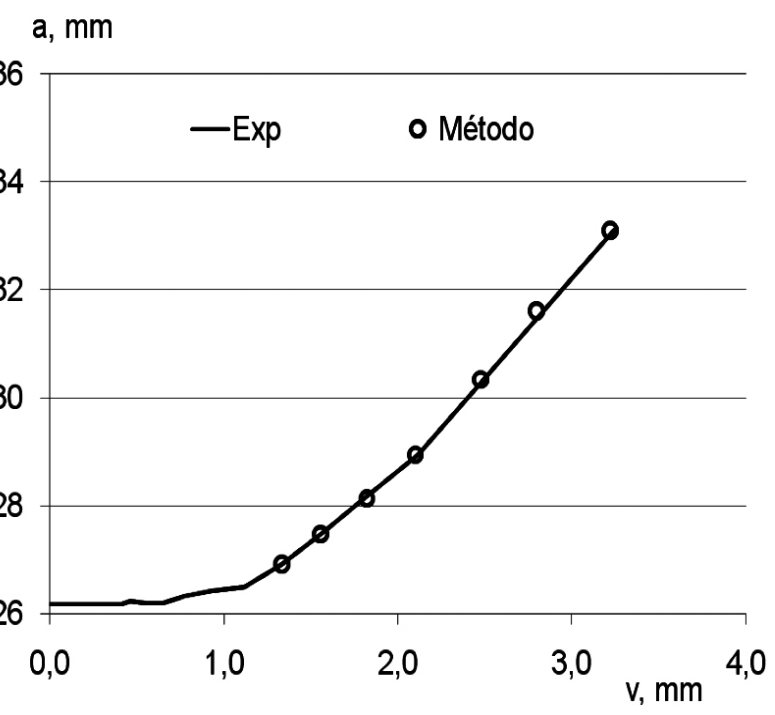

Figura 5. Curva a-v experimental, y valores de tamaño de grieta obtenidos con el método.

Figure 5. Experimental a-v curve and crack sizes obtained with the method.

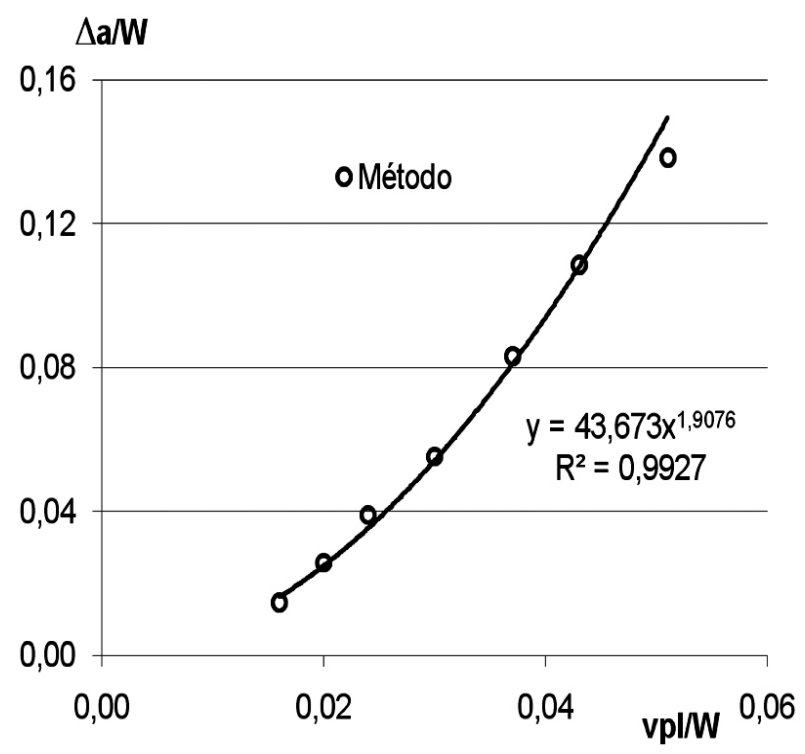

Figura 6. La ley de crecimiento de grieta obtenida con el método.

Figure 6. Crack growth law obtained with the method.

\section{EL MODELO NUMÉRICO PARA LA PROBETA C(T) DE A508}

Con objeto de verificar el método del intercepto presentado en el apartado anterior, se realizó una modelización por elementos finitos, usando el programa ADINA. Para ello, se modelizó el caso experimental, en que hay crecimiento de grieta ("a variable") y siete casos de tamaño de grieta constante ("a constante"). En estos ocho casos, la respectiva probeta $\mathrm{C}(\mathrm{T})$ se modelizó como bidimensional.

La probeta "a variable", a la que se impuso la modalidad de propagación de grieta del programa (mediante liberación de nodos), tenía $a_{0} / W=0,52$. La liberación programada de nodos que emula el crecimiento de grieta, se realizó mediante control por desplazamiento en el punto de aplicación de la carga (Figs. 7 y 8), siendo necesario ingresar al programa una relación entre el avance de grieta, $\Delta a$, y el desplazamiento total impuesto, $\Delta$. Esta relación es la misma que se puede apreciar en la figura 5 , dada por la curva experimental $a-v$ para la probeta de A508.

En las modelizaciones del tipo "a constante", las probetas fueron sometidas a un desplazamiento en el punto de aplicación de la carga, pero sin hacer crecer la grieta. Los valores del desplazamiento prescrito, para cada una de los siete modelos del tipo "a constante", son los mismos desplazamientos que adopta la probeta real, en cada uno de los puntos "16" a "22", respectivamente, acorde con la figura 4. 


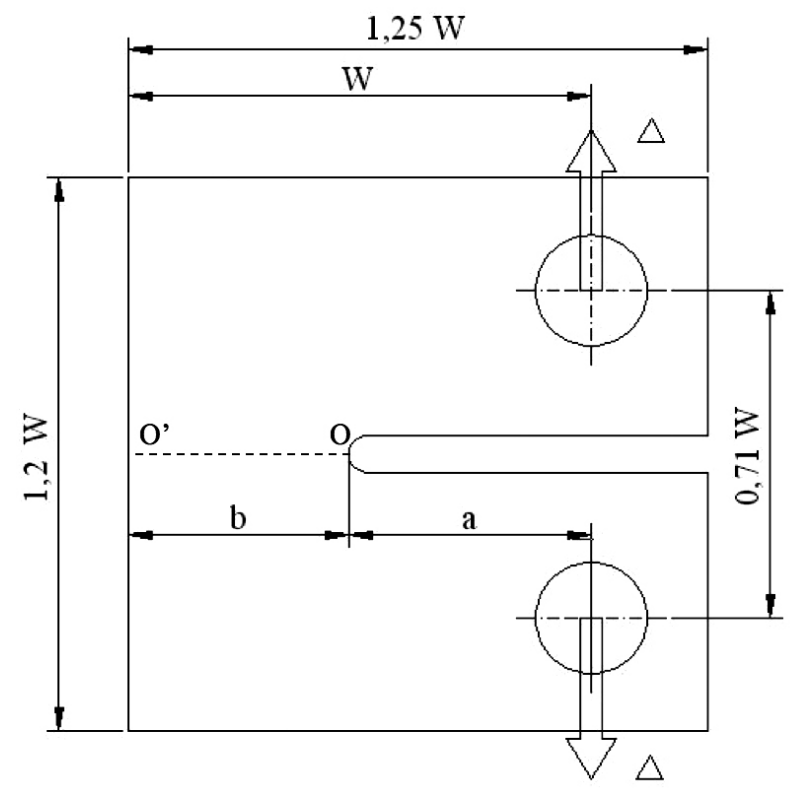

Figura 7. Esquema de la probeta $\mathrm{C}(\mathrm{T})$.

Figure 7. Schematics of the $C(T)$ specimen.

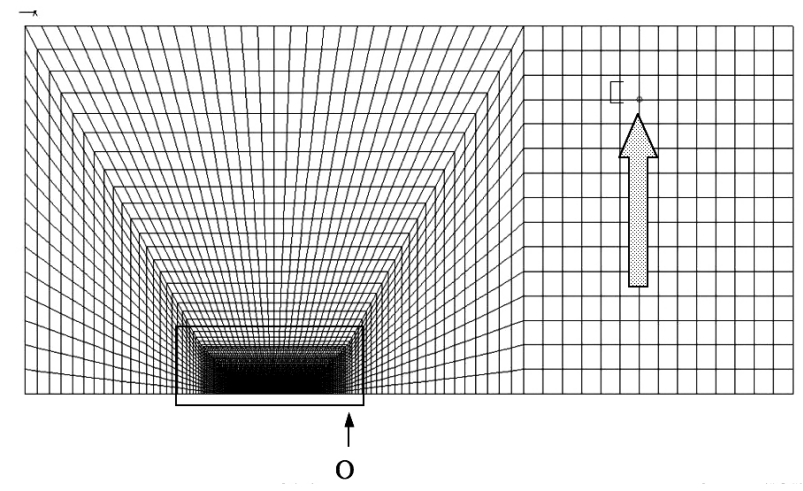

Figura 8. Malla de la probeta $C(T)$. La punta de la grieta se muestra por la flecha (“O”), en tanto el sector enmarcado se muestra en la figura 9.

Figure 8. Mesh of the $C(T)$ specimen. The crack tip is shown by the arrow ("O"), whereas the area within the box is shown in figure 9.

Debido a la simetría de la probeta $\mathrm{C}(\mathrm{T})$, sólo se modelizó la mitad de ella. La malla utilizada tiene 9070 elementos 2D de cuatro nodos, con un total de 9160 nodos (Fig. 8). Para facilitar la simulación de crecimiento de grieta, se uso una malla fina y uniforme en la vecindad de la punta de la grieta, como muestra la figura 9. La dimensión del elemento más pequeño localizado en esta región es $50 \mu \mathrm{m}$. El material se consideró como elasto-plástico multi-lineal y se asumió condición de deformación plana.

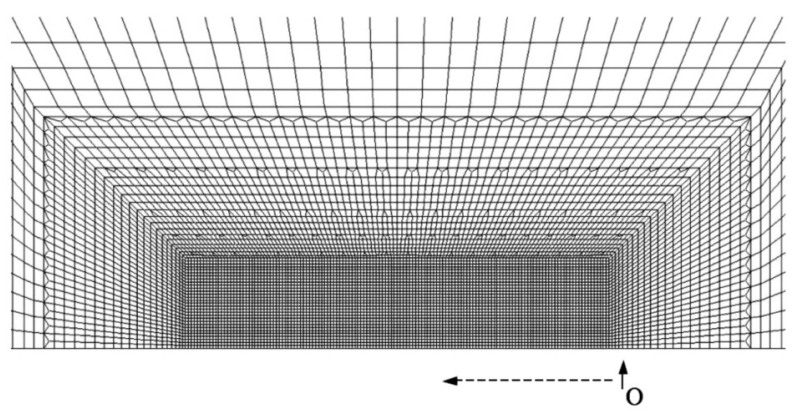

Figura 9. Detalle de la malla en la vecindad de la punta de la grieta, "O". La extensión estable de grieta se produce desde "O" hacia la izquierda, por liberación de nodos.

Figure 9. Mesh detail in the vicinity of the crack tip, "O". The stable crack extension is generated from "O" to the left, by node release.

Los resultados de la modelización se pueden ver en la figura 10. En esta figura se ha representado el resultado $P-v$ del modelo bidimensional (línea continua) contra los datos experimentales, $(\bullet)$, advirtiéndose una excelente relación experimento-modelo. Más relevante, sin embargo, es el hecho que cada una de las siete curvas del tipo "a constante", para los tamaños de grieta entre 27,0 y $33,0 \mathrm{~mm}$, interceptan a la curva "a variable" en el punto correspondiente a la tríada " $P_{i}, v_{i}, a_{i}$ ". En este contexto, el símil con la figura 4 es extraordinario.

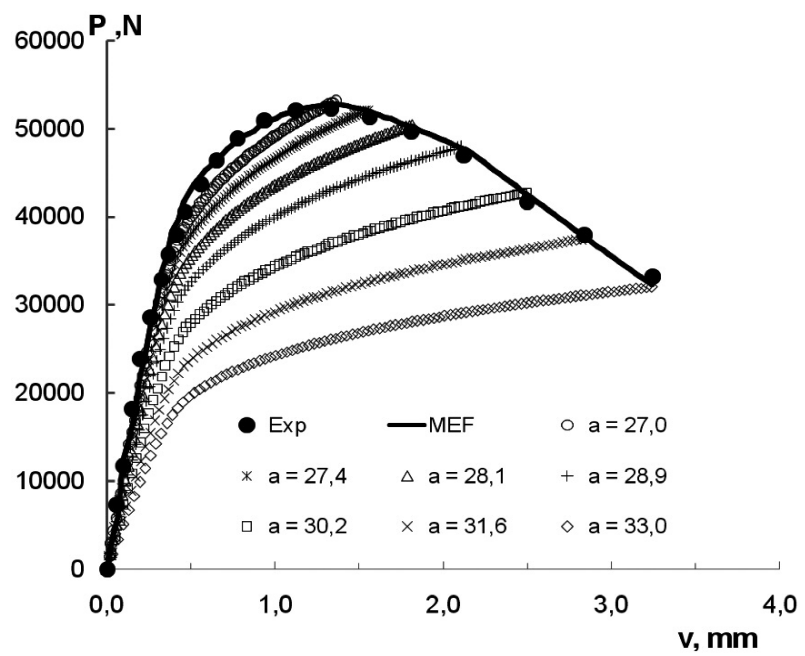

Figura 10. Resultados fuerza-desplazamiento de la modelación de la probeta $\mathrm{C}(\mathrm{T})$ por elementos finitos.

Figure 10. The $C(T)$ specimen forcedisplacement results obtained with the finite element model. 


\section{LA CURVA J-R DERIVADA DEL MÉTODO PROPUESTO}

En los capítulos precedentes, se ha demostrado que la tríada " $P_{i}, v_{i}, a_{i}$ " obtenida con el método del intercepto, es igual (o muy similar, dentro de los márgenes del error experimental) a aquella propia del experimento, para cada dato $P-v-a$ existente. La figura 5 avala esta aseveración, al obtener con el método propuesto valores de tamaño de grieta vs. desplazamiento, similares a los obtenidos experimentalmente. Más aún, la modelación numérica de curvas $P-v$ del tipo "a constante" permitió confirmar que, al dar como dato un valor de desplazamiento $v_{j}$, tal que, $v_{c}$ $<v_{j}<v_{f}$, y de tamaño de grieta $a j$, tal que $a_{o}<a_{j}<$ $a_{f}$, entonces, el resultado esperado es $P_{j}$, un valor de fuerza que estará sobre la curva $P-v$ experimental, del tipo "a variable". Los resultados de las figuras 4 y 10 confirman esta propuesta.

Si los valores $P-v$-a generados con el método propuesto son similares a los obtenidos experimentalmente, entonces, la curva J-R construida con el método también debe ser similar a la curva $J-R$ experimental. La figura 11 muestra la curva original para la probeta $\mathrm{C}(\mathrm{T})$ de A $508(\circ)$, aquella, obtenida con el modelo DZL $(\Delta)^{[3 \text { y } 4]}$, y valores obtenidos con el

\section{$\mathrm{J}, \mathrm{N} / \mathrm{mm}$}

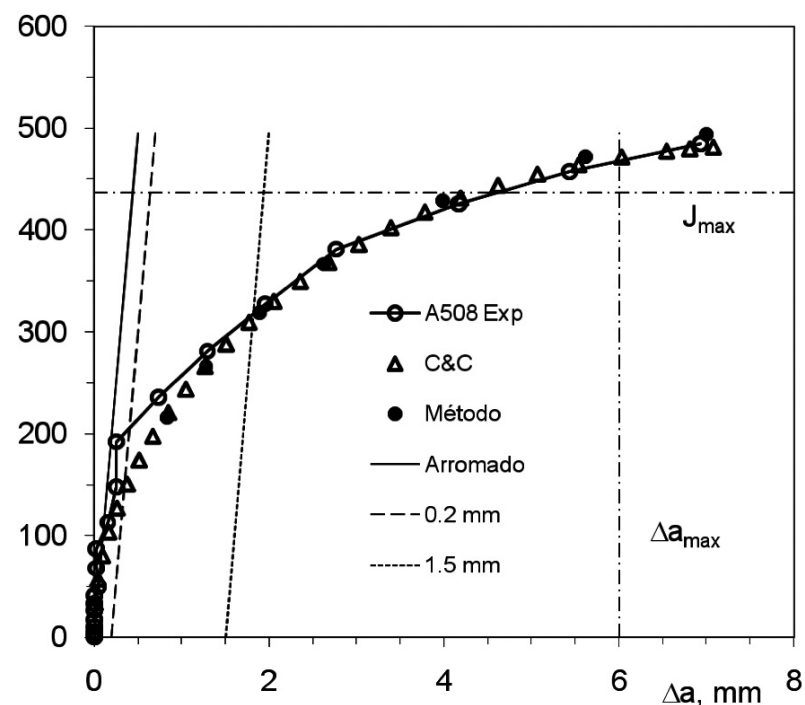

Figura 11. Valores de $J$ vs $\Delta a$ obtenidos con el método, comparados con la curva J-R experimental y con aquella obtenida con los formatos c\&C.

Figure 11. J vs $\Delta a$ values obtained with the method, compared with the experimental J-R curve and with that obtained with the C\&C formats. método propuesto $(\bullet)$. En esta figura se advierte que el método del intercepto da resultados similares (si no iguales) a los obtenidos con la Norma ${ }^{[3}$ y 4$]$.

\section{COMENTARIOS FINALES}

El método alternativo de construcción de la curva $J-R$ propuesto por Donoso, Zahr y Landes (DZL) [3 y 4] utilizaba la noción de una "ley de crecimiento de la grieta", relacionando el monto de extensión estable de la grieta, $\Delta a$, normalizada por $W$, con el desplazamiento plástico normalizado, $v_{p l} / W$. Esta ley de crecimiento de grieta se propuso en la forma de una relación de tipo ley de potencia con dos parámetros: un coeficiente, $l_{o}$, y un exponente, $l_{1}$.

De estos dos parámetros, en la propuesta inicial de DZL, el coeficiente $l_{o}$ se sustituía en función de $l_{1}$, y de las coordenadas de un punto de calibración conocido, como es el punto final del ensayo (punto "F" en la figura 1): desplazamiento total, $v_{f}$, fuerza, $P_{f}$, y tamaño final de grieta, $a_{f}$. De este modo, el modelo de la ley de crecimiento de grieta tenía sólo un parámetro ajustable: el exponente $l_{1}$. El valor de $l_{1}$ que mejor acomodaba los valores experimentales de fuerza y desplazamiento (es decir, permitía reproducir mejor la curva experimental $P-v$ ) era 2,0. Este valor se usó en un par de ejemplos de datos $P$ $v$ en probetas 1T-C(T) de aceros A 508 y A 533B con valores conocidos de extensión de grieta a lo largo del ensayo, dando un muy buen ajuste entre la curva experimental $P-v$ y la curva "a variable" C\&C [3 y 4].

A partir de estos resultados exitosos con el método señalado, se propuso que un tratamiento analítico de tanto la curva $P-v$ como la curva $J-\Delta a$ sería posible, entonces, incluso bajo circunstancias en las cuales los únicos datos disponibles fuesen los pares $P-v$, más los valores inicial y final del tamaño de la grieta, sin medir simultáneamente los valores de extensión de la grieta con el ensayo de fractura. Para ello, sin embargo, el concepto de ley de crecimiento de grieta necesitaba un fundamento analítico más sólido y una prueba empírica (en este caso, numérica) de su viabilidad. Esto, ha constituído el núcleo central de este trabajo.

En efecto, el método presentado aquí, llamado "método del intercepto", es equivalente a aquél utilizado en trabajos previos ${ }^{[3}$ y 4$]$, si se acepta que el punto de calibración, " $F$ ", puede ser cualquiera de los puntos designados con los números 16 al 22 en la figura 4. El método presentado en este trabajo se basa en el hecho de que las Ecuaciones de Formato Común y Conciso $(\mathrm{C} \& \mathrm{C})$ pueden generar curvas $P-v$ "a constante", de tamaños de grieta conocidos, 
con valores entre el inicial, $a_{\circ}$, y el tamaño final de grieta medido físicamente, $a_{f}$. El intercepto de estas curvas del tipo "a constante" con la curva experimental, dará lugar a valores de desplazamiento total y fuerza para los cuales la curva experimental tendrá un tamaño de grieta igual al de la curva " $a$ constante" que la intercepta. Con estos interceptos se podrá calcular el valor del desplazamiento plástico en cada punto, y mediante el enfoque $\mathrm{C} \& \mathrm{C}$, se podrá disponer de un conjunto completo de valores de extensión de grieta, donde originalmente no los hay.

La ley de crecimiento de grieta desarrollada previamente ${ }^{[3 \text { y } 4]}$, es un complemento ideal, pero eventual del "método del intercepto". De hecho, puede ser utilizada para aumentar la cantidad de datos de tamaños de grieta generados con el método, con el objeto de producir una curva $J-R$ con mayor número de puntos (como la curva denominada $C \& C$ en la figura 11). El gran mérito del "método del intercepto" es obtener valores de tamaño de grieta donde no los hay disponibles. De esta manera, la construcción de una curva $J-R$ será posible ahora a partir de datos $P-v$ que sólo tengan como complemento el tamaño inicial y el tamaño final de la grieta.

\section{Agradecimientos}

Este trabajo contó con el apoyo del Proyecto FONDECYT 1060415, y del Proyecto USM/DGIP 21.06.21.

\section{REFERENCIAS}

[1] ASTM E 1820-08, American Society for Testing and Materials, 2008.

[2] B.P. Pehrson, y J.D. Landes, Fatigue Fract. Eng.Mater. Struct. 30 (2006) 73-86.

[3] J.R. Donoso, J. Zahr y J.D. Landes, Fatigue and Fracture Mechanics, $34^{\text {th }}$ Volume, ASTM STP 1461, S.R. Daniewicz, J.C. Newman y K.-H. Schwalbe, Eds., ASTM International, West Conshohocken, PA, 2005, pp. 323-339

[4] J.R. Donoso, J.Zahr y J.D. Landes, Rev. Metal. Madrid 41 (2005) 401-480.

[5] J.R. Donoso, K. Vásquez y J.D. Landes, J. ASTM Int. (JAI) 5 (2008) 1-15.

[6] J.R. Donoso y J.D. Landes, Fatigue and Fracture Mechanics: $30^{\text {th }}$ Volume, ASTM 1360, P.C. Paris and K.L. Jerina, Eds., ASTM International, West Conshohocken, PA, 2000, pp. 34-50.

[7] J.R. Donoso y J.D. Landes, Eng. Fract. Mech. 47 (1994) 619-628.

[8] J.R. Donoso y J.D. Landes, Fatigue and Fracture Mechanics: $32^{\text {nd }}$ Volume, ASTM 1406, R. Chona. Ed., ASTM International, West Conshohocken, PA, 2001, pp. 261-278.

[9] J.R. Donoso y J.D. Landes, ECF17, $17^{\text {th }}$ Europ. Conf. Fracture, Brno, 2008, pp. 539-546.

[10] H.A. Ernst, P.C. Paris y J.D. Landes, Fracture Mechanics; Thirteenth Conf., ASTM STP 743, Richard Roberts, Ed., ASTM International, 1981, pp. 476-502.

[11] J.R. Donoso, A. Ortiz, , y F. Labbé, Rev. Metal. Madrid 39 (2003) 357-366. 